\title{
TITLE:
}

\section{Occupation of a resonant level coupled to a chiral Luttinger liquid}

$\operatorname{AUTHOR}(S)$ :

Furusaki, A; Matveev, KA

CITATION:

Furusaki, A ...[et al]. Occupation of a resonant level coupled to a chiral Luttinger liquid. Physical Review Letters 2002, 88(22): 226404.

ISSUE DATE:

2002-06-03

URL:

http://hdl.handle.net/2433/50455

RIGHT:

Copyright 2002 American Physical Society 


\title{
Occupation of a Resonant Level Coupled to a Chiral Luttinger Liquid
}

\author{
A. Furusaki ${ }^{1}$ and K. A. Matveev ${ }^{2}$ \\ ${ }^{1}$ Yukawa Institute for Theoretical Physics, Kyoto University, Kyoto 606-8502, Japan \\ ${ }^{2}$ Department of Physics, Duke University, Box 90305, Durham, North Carolina 27708
}

(Received 17 October 2001; published 20 May 2002)

\begin{abstract}
We consider a resonant level coupled to a chiral Luttinger liquid which can be realized, e.g., at a fractional quantum Hall edge. We study the dependence of the occupation probability $n$ of the level on its energy $\varepsilon$ for various values of the Luttinger-liquid parameter $g$. At $g<1 / 2$, a weakly coupled level shows a sharp jump in $n(\varepsilon)$ at the Fermi level. As the coupling is increased, the magnitude of the jump decreases until $\sqrt{2 g}$, and then the discontinuity in $n(\varepsilon)$ disappears. We show that $n(\varepsilon)$ can be expressed in terms of the magnetization of a Kondo impurity as a function of magnetic field.
\end{abstract}

\section{DOI: 10.1103/PhysRevLett.88.226404}

The transport of electrons in low-dimensional structures is strongly affected by electron-electron interactions. This effect is particularly strong in one-dimensional (1D) systems, where the interacting electrons form the so-called Tomonaga-Luttinger liquid [1]. The main feature of this system is the power-law suppression of the tunneling density of states at low energies. This effect has been recently observed experimentally in 1D electron systems realized in quantum Hall edges [2], carbon nanotubes [3,4], and quantum wires [5].

A generic property of mesoscopic devices is the presence of imperfections that affect the transport of electrons. The most important are the defects that form energy levels near the Fermi level and give rise to a strong scattering of electrons. In this paper, we consider a single level coupled to a 1D system exhibiting Luttinger liquid behavior. The level can be caused either by a random impurity or by an artificially created quantum dot near the $1 \mathrm{D}$ system. Recently, electron transport through a resonant level coupled to two Luttinger-liquid leads was studied both experimentally $[5,6]$ and theoretically [7-9].

In contrast to the previous theoretical work [7-9], we study a resonant level coupled to a single 1D conductor. The quantity of interest is the occupation probability $n$ of the level as a function of its energy $\varepsilon$ (measured from the Fermi level). Clearly, at large positive energy $\varepsilon$ the level is empty, $n=0$, and at large negative energy it is filled, $n=1$. If the level is coupled to a Fermi-liquid lead, $n(\varepsilon)$ changes continuously from 0 to 1 when the energy $\varepsilon$ is within the level width $\Gamma$ from the Fermi level:

$$
n(\varepsilon)=\frac{1}{2}-\frac{1}{\pi} \arctan \frac{\varepsilon}{\Gamma} .
$$

We show below that the dependence $n(\varepsilon)$ is modified significantly in the case of coupling to a Luttinger-liquid lead. Most importantly, if the interactions are strong enough, $n(\varepsilon)$ has a discontinuity at $\varepsilon=0$.

We model the level coupled to a 1D conductor by the Hamiltonian $H=H_{0}+H_{t}$, where

$$
H_{0}=\varepsilon a^{\dagger} a+\frac{\hbar v}{4 \pi} \int_{-\infty}^{\infty}\left(\frac{d \varphi}{d x}\right)^{2} d x,
$$

PACS numbers: 71.10.Pm, 72.10.Fk, 73.21.Hb, 73.43.Jn

$$
H_{t}=t\left(a^{\dagger} \psi+\psi^{\dagger} a\right), \quad \psi=\sqrt{\frac{D}{2 \pi \hbar v}} e^{i \varphi(0) / \sqrt{g}} .
$$

The first term in Eq. (2) describes the resonant level with energy $\varepsilon$; the fermion operator $a^{\dagger}$ creates an electron in this state. The second term in Eq. (2) describes the Tomonaga-Luttinger liquid in the lead in terms of a chiral boson field $\varphi(x)$ with commutation relations $[\varphi(x), \varphi(y)]=i \pi \operatorname{sgn}(x-y)$. The chiral Luttinger liquid model is the most natural description of fractional quantum Hall edge states [10]. In the case of a nonchiral 1D system coupled to the level at only a single point $x=0$, one can neglect the "odd" bosonic modes and convert [11] the Hamiltonian to the chiral form (2). The Hamiltonian $H_{t}$ describes the tunneling of the electron between the level and the point $x=0$ in the $1 \mathrm{D}$ system. We use the standard bosonized expression for the annihilation operator $\psi$ of an electron in the chiral Luttinger liquid in terms of the field $\varphi(x)$, the Luttinger-liquid parameter $g$, the bandwidth $D$, and the Fermi velocity $v$. In this paper, we consider only the case of spinless electrons. Many of our results are not sensitive to this assumption. Also, in the case of tunneling into edge states of the quantum Hall system with filling factor $g<1$, all electrons are spin polarized by the magnetic field. In other cases, the level can be polarized by applying the magnetic field.

We first calculate the occupation probability $n(\varepsilon)$ in the second-order perturbation theory in $H_{t}$. At $\varepsilon>0$ the result has the natural form,

$$
n(\varepsilon)=\frac{\Gamma}{\pi \nu_{1}(0)} \int_{0}^{\infty} \frac{\nu_{g}(\omega)}{(\omega+\varepsilon)^{2}} d \omega .
$$

Parameter $\Gamma=t^{2} / 2 \hbar v$ has the physical meaning of the level width in the Fermi-liquid case $g=1$. The tunneling density of states $\nu_{g}(\omega)$ of the Tomonaga-Luttinger liquid has the well-known form

$$
\nu_{g}(\omega)=\frac{1}{2 \pi \hbar v \Gamma\left(g^{-1}\right)}\left(\frac{|\omega|}{D}\right)^{(1 / g)-1} e^{-|\omega| / D},
$$

where $\Gamma(x)$ is the gamma function. Because of the electron-hole symmetry of the problem, the occupation 
probability at negative energies can be found as $n(\varepsilon)=1-n(-\varepsilon)$. At $g=1$, Eq. (4) coincides with expansion of the Fermi-liquid result (1) at $\varepsilon \gg \Gamma$.

At $g>1 / 2$, the integral in Eq. (4) diverges at $\varepsilon \rightarrow 0$. This means that, in order to find $n(\varepsilon)$ near the Fermi level, one has to sum up the whole series of the perturbation theory in $t$. On the other hand, at $g<1 / 2$ the integral is convergent at all $\varepsilon$; i.e., for a weakly coupled level the second-order perturbation theory is sufficient to describe $n(\varepsilon)$ at any energy. For instance, at $\varepsilon \rightarrow+0$, we find

$$
n(+0)=\frac{g^{2}}{\pi(1-g)(1-2 g)} \frac{\Gamma}{D} .
$$

At $\varepsilon \ll D$ the occupation probability approaches its limit (6) following a power-law dependence:

$$
n(+0)-n(\varepsilon)= \begin{cases}\frac{\Gamma}{\pi D}\left|\Gamma\left(\frac{2 g-1}{g}\right)\right|\left(\frac{\varepsilon}{D}\right)^{(1 / g)-2} & \frac{1}{3}<g<\frac{1}{2} \\ \frac{\Gamma}{\pi D} \frac{\varepsilon}{D} \ln \frac{D}{\varepsilon} & g=\frac{1}{3} \\ \frac{2 g^{3}}{(1-g)(1-2 g)(1-3 g)} \frac{\Gamma}{\pi D} \frac{\varepsilon}{D} & g<\frac{1}{3} .\end{cases}
$$

Since $n(+0) \neq n(-0) \equiv 1-n(+0)$, the system reaches two different ground states at $\varepsilon \rightarrow \pm 0$, and the occupation probability $n(\varepsilon)$ experiences a finite discontinuity at the Fermi level: $\Delta \equiv n(-0)-n(+0)$. The divergence of the perturbation theory at $g>\frac{1}{2}$ indicates that in this regime the coupling of the level to the lead is a relevant perturbation that should lift the degeneracy of the ground state at $\varepsilon=0$, resulting in continuous $n(\varepsilon)$. This can be shown formally using the mapping to the Kondo problem discussed below.

As the coupling $\Gamma$ of the level to the 1D system grows, the magnitude of the jump $\Delta=1-2 n(+0)$ at $g<\frac{1}{2}$ decreases. We will now show that at a sufficiently strong coupling the jump disappears. Let us consider a semiinfinite quantum wire in the region $x>0$ with a scatterer at point $x=L$. The system is described by the TomonagaLuttinger model with a scatterer:

$$
H=\frac{\hbar v}{2 \pi} \int_{0}^{\infty}\left[\frac{1}{g}\left(\frac{d \theta}{d x}\right)^{2}+g\left(\frac{d \phi}{d x}\right)^{2}\right] d x-w \cos (2 \pi N) .
$$

Here $N=\left[k_{F} L+\theta(L)\right] / \pi$ is the number operator of electrons in the region $0<x<L$. The fields $\theta$ and $\phi$ satisfy the commutation relation $[\theta(x), \phi(y)]=(i \pi / 2)[1+$ $\operatorname{sgn}(x-y)]$ and the boundary condition $\theta(x=0)=0$.

If the scatterer is very strong, $w \rightarrow \infty$, it effectively cuts the region $0<x<L$ from the wire. This region becomes a quantum dot, coupled to the rest of the wire by tunneling through the barrier created by the scatterer. At large $w$ the field $\theta$ at point $x=L$ is pinned at one of the values $\theta(L)=\pi m-k_{F} L$, where integer $m$ is the number of electrons in the dot. The ground state energy of the dot is then $E_{m}=(\hbar v / 2 \pi g L)\left(\pi m-k_{F} L\right)^{2}$. For a given $L$, the number of electrons in the dot that minimizes $E_{m}$ is the integer nearest to $k_{F} L / \pi$. One can easily check that, at $k_{F} L / \pi=M+\frac{1}{2}-\frac{g L}{\pi \hbar v} \varepsilon$ with integer $M$ and positive $\varepsilon$, the ground state of the dot has
$m=M$ electrons, and the energy of adding $(M+1)$ st electron is $E_{M+1}-E_{M}=\varepsilon$. Thus, at large $w$ the Hamiltonian (8) is equivalent to the model (2), (3) of a resonant level coupled to a 1D system [12].

One can now increase the coupling by lowering $w$. In the limit of very strong coupling, $w \rightarrow 0$, the scattering term in the Hamiltonian (8) can be accounted for in first-order perturbation theory. In an infinite 1D system, the thermal average of the scattering potential shows power-law behavior at low temperatures as $(T / D)^{g} w \cos \left(2 k_{F} L\right)$. However, if the distance $L$ from the scatterer to the end of the wire is finite, the renormalization of the scattering potential is cut off at the low energy scale equal to the level spacing in the dot, $\sim \hbar v / L$. Although the renormalization of the scattering potential in a semi-infinite wire may be large, it remains finite, and, at sufficiently small $w$, one can use the first-order result $\delta E_{0} \sim(\hbar v / L D)^{g} w \cos \left(2 k_{F} L\right)$. Thus, at strong coupling the energy is an analytic function of $k_{F} L$ which shows no singularities at degeneracy points $k_{F} L=\pi\left(M+\frac{1}{2}\right)$, where $\varepsilon=0$. The analyticity of $E_{0}(\varepsilon)$ means that the ground state is nondegenerate, and the occupation probability $n(\varepsilon)=\partial E_{0} / \partial \varepsilon$ [see Eqs. (2) and (3)] is continuous.

From the perturbative calculations in the weak- and strong-coupling limits, we deduce a schematic phase diagram, Fig. 1. The occupation probability $n(\varepsilon)$ is a continuous function of $\varepsilon$ at $g>\frac{1}{2}$ (including the Fermi-liquid case $g=1$ ) and also at $g<\frac{1}{2}$, if the coupling to the lead is sufficiently strong. On the other hand, if $g<\frac{1}{2}$ and the coupling is weak, $n(\varepsilon)$ has a discontinuity at $\varepsilon=0$. As the coupling is increased at $g<\frac{1}{2}$, the discontinuity $\Delta$ decreases until the phase boundary is reached, above which $\Delta=0$. We now show that $\Delta$ is not a continuous function of the coupling strength and that just below the boundary $\Delta=\sqrt{2 g}$.

Let us consider a level relatively weakly coupled to the lead with $g<\frac{1}{2}$. The presence of a jump in the occupation probability $n(\varepsilon)$ means that at $\varepsilon=0$ the ground state of the system is degenerate. One could expect that tunneling term (3), which couples the two degenerate states by

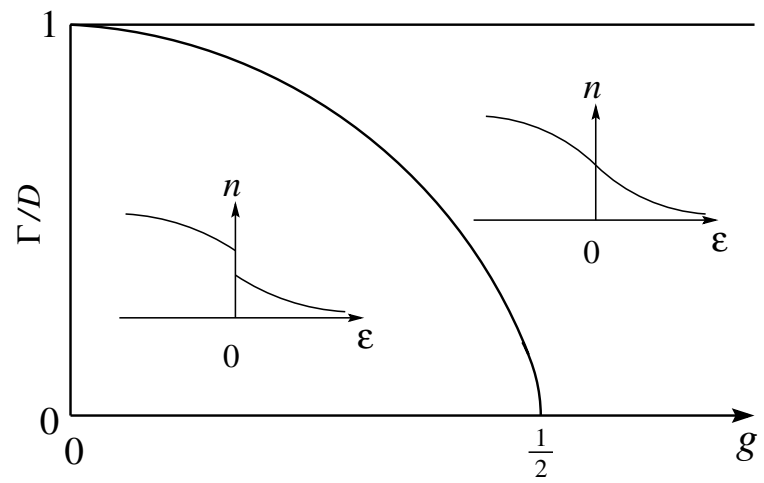

FIG. 1. Schematic phase diagram. Insets show the behavior of the occupation probability $n(\varepsilon)$. The discontinuity occurs at $g<\frac{1}{2}$ and weak enough effective coupling $\Gamma / D$. 
moving an electron from the level to the lead, would lift the degeneracy. This does not always happen because the tunneling of an electron into a Tomonaga-Luttinger liquid is suppressed at low energies, leading to the renormalized level width $\tilde{\Gamma}(\varepsilon) \propto \varepsilon^{\gamma}$. Thus, at $\gamma>1$ the coupling is irrelevant, as it is much smaller than the level separation $\varepsilon$. In the opposite case of $\gamma<1$, the width is much larger than the level separation at $\varepsilon \rightarrow 0$, so the degeneracy of the ground state is lifted. Thus, at the phase boundary in Fig. 1 the exponent $\gamma=1$.

At $t \rightarrow 0$, the suppression of tunneling manifests itself in the power-law behavior of the density of states (4). Thus, the exponent $\gamma=\frac{1}{g}-1$, and the condition $\gamma>1$ of the degenerate ground state reproduces our earlier result $g<\frac{1}{2}$. At $t \neq 0$ the virtual hopping processes smear the quantization of the charge, and at low energies the perturbation $H_{t}$ transfers only a fraction $\Delta$ of an electron charge into the lead. Mathematically this can be accounted for by a renormalized coupling term $\tilde{H}_{t} \propto t\left[a^{\dagger} e^{i \varphi(0) \Delta / \sqrt{g}}+\right.$ H.c.], cf. Eq. (3). Thus the exponent of the tunneling density of states becomes $\gamma=\frac{\Delta^{2}}{g}-1$ when only a fraction $\Delta$ of the electron charge is transferred. At the phase boundary the condition $\gamma=1$ is then equivalent to $\Delta=\sqrt{2 g}$.

The phase diagram [Fig. 1] and the critical value of the jump $\Delta=\sqrt{2 g}$ at the phase boundary are the main results of this paper [13].

One can also study how $n(\varepsilon)$ approaches the discontinuity at $\varepsilon=0$ anywhere below the phase boundary in Fig. 1 . The discussion is essentially identical to the one leading to Eq. (7) at $t \rightarrow 0$. By substituting $\nu(\omega) \propto \omega^{\gamma}$ instead of (5) into (4), we find at small positive $\varepsilon$ and for $\Delta>\sqrt{2 g}$,

$$
\frac{1-\Delta}{2}-n(\varepsilon) \propto \varepsilon^{\min \left\{\left(\Delta^{2} / g\right)-2,1\right\}} .
$$

Additional support for the above results is found by mapping our model to two well-known problems: a two-level system with Ohmic dissipation and the Kondo problem. Let us define a unitary operator $U_{1}=\exp \left[i \varphi(0) S^{z} / \sqrt{g}\right]$, where $S^{z}=a^{\dagger} a-1 / 2$. Unitary transformation of the Hamiltonian (2), (3) yields the Hamiltonian of the two-level system:

$$
U_{1}^{\dagger} H U_{1}=H_{0}+t \sqrt{\frac{2 D}{\pi \hbar v}} S^{x}-\frac{\hbar v}{\sqrt{g}} S^{z} \frac{d \varphi(0)}{d x}+\text { const, }
$$

where $S^{x}=\left(a+a^{\dagger}\right) / 2$. The second term describes hopping of a particle between the two levels, $S^{z}= \pm \frac{1}{2}$, and the third term is the coupling to harmonic oscillators with dissipation strength $\alpha=1 / 2 g$ [14]. It is known that there is a quantum phase transition between a phase where a particle is localized in one of the two levels and another phase where it is delocalized. In the limit $t \rightarrow 0$, the transition occurs at $\alpha=1$ [14], in agreement with our weakcoupling result [15]. Our analysis suggests that, as the coupling $t$ is increased in the localized phase, the difference $\Delta$ of the averages $\left\langle S^{z}\right\rangle$ over the two ground states at $\varepsilon=0$ will decrease from 1 to $\sqrt{2 g}=\alpha^{-1 / 2}$, after which the system will suddenly delocalize, $\Delta=0$. To the best of our knowledge, only a brief discussion [16] can be found in the literature on the $\varepsilon$ dependence of the ground-state average $\left\langle S^{z}\right\rangle$ at $\alpha=1-\eta$, and the result is consistent with $\Delta=\alpha^{-1 / 2}$ to linear order in $\eta$.

To understand the connection to the Kondo problem, let us introduce another operator $U_{2}=$ $U_{1} \exp \left[-i \sqrt{2} \varphi(0) S^{z}\right]$. Unitary transformation yields

$$
\begin{aligned}
U_{2}^{\dagger} H U_{2}= & H_{0}+t \sqrt{\frac{D}{2 \pi \hbar v}}\left(S^{+} e^{i \sqrt{2} \varphi(0)}+S^{-} e^{-i \sqrt{2} \varphi(0)}\right) \\
& -\hbar v\left(\frac{1}{\sqrt{g}}-\sqrt{2}\right) S^{z} \frac{d \varphi(0)}{d x}+\text { const, }
\end{aligned}
$$

where $S^{+}=a^{\dagger}$ and $S^{-}=a$. Equation (10) is also obtained by bosonizing the anisotropic Kondo model describing electrons scattered by a spin- $-\frac{1}{2}$ impurity,

$$
\begin{aligned}
H_{K}= & \int \epsilon_{k} a_{k \mu}^{\dagger} a_{k \mu} d k+\iint J_{i} S^{i} \sigma_{\mu \nu}^{i} a_{k \mu}^{\dagger} a_{k^{\prime} \nu} d k d k^{\prime} \\
& +\varepsilon S^{z},
\end{aligned}
$$

where $\epsilon_{k}=\hbar v k, \sigma^{i}$ is the Pauli matrix, and summation over repeated indices is assumed $(\mu, \nu=\uparrow, \downarrow$ and $i=$ $x, y, z)$. The Kondo couplings $J_{i}$ are given by

$$
J_{\perp}=t \sqrt{\frac{2 \pi \hbar v}{D}}, \quad J_{z}=2 \pi \hbar v\left(1-\frac{1}{\sqrt{2 g}}\right),
$$

where $J_{x}=J_{y}=J_{\perp}$. The occupation probability $n(\varepsilon)$ is directly related to the magnetization $\mathcal{M}$ of the impurity spin $S$ coupled to the Zeeman field $\varepsilon: n(\varepsilon)=\mathcal{M}(\varepsilon)+$ $1 / 2$. The phase diagram [Fig. 1] is easily understood from the renormalization-group flow diagram of the Kondo problem. The phase of a smooth $n(\varepsilon)$ curve corresponds to the antiferromagnetic Kondo coupling, $J_{z}>-\left|J_{\perp}\right|$. In this case the impurity spin is completely screened as the magnetic field $\varepsilon$ is turned off: $\mathcal{M}( \pm 0)=0$. At $g-$ $\frac{1}{2} \ll 1$ in the weak-coupling limit, $\Gamma \ll D$, one can easily obtain $n(\varepsilon)$ from the Bethe ansatz results [17] for $\mathcal{M}(\varepsilon)$.

The phase of a discontinuous $n(\varepsilon)$ curve in Fig. 1 corresponds to the ferromagnetic Kondo case, $J_{z}<-\left|J_{\perp}\right|$, where the spin flip coupling $J_{\perp}$ is renormalized to zero. One might then naively expect a full moment to appear: $\mathcal{M}=-\operatorname{sgn}(\varepsilon) / 2$. This is not correct, however, as we have seen in Eqs. (6) and (7). In the weak-coupling regime, we may use one-loop scaling equations for $j_{i}=J_{i} / 2 \pi \hbar v$ :

$$
\frac{d j_{\perp}}{d l}=2 j_{\perp} j_{z}, \quad \frac{d j_{z}}{d l}=2 j_{\perp}^{2}, \quad \frac{d \mathcal{M}}{d l}=-2 j_{\perp}^{2} \mathcal{M},
$$

where $d l=-d \ln D$. For $\left|j_{\perp}\right| \leq-j_{z}$ we integrate them to $l=\ln (D / \varepsilon)$ with initial condition $\mathcal{M}=-1 / 2$. We find $2 \mathcal{M}(\varepsilon)=-\exp \left(j_{z}-j_{z}^{*}\right)$, where

$$
\frac{j_{z}^{*}}{c}=\frac{j_{z}-c+\left(c+j_{z}\right)(\varepsilon / D)^{4 c}}{c-j_{z}+\left(c+j_{z}\right)(\varepsilon / D)^{4 c}}, \quad c=\sqrt{j_{z}^{2}-j_{\perp}^{2}},
$$

in agreement with Ref. [16]. To lowest order in $\frac{1}{2}-g$, this one-loop result is consistent with Eq. (9). In particular, at the transition point $j_{\perp}=j_{z}$, the magnitude of the 
magnetization jump $\Delta=\mathcal{M}(-0)-\mathcal{M}(+0)=1+j_{z}$ agrees with our conjecture $\sqrt{2 g}=\left(1-j_{z}\right)^{-1}$ in the first order in $j_{z}$. Approach to the zero-field value is logarithmically slow at the transition point: $\mathcal{M}(\varepsilon)-\mathcal{M}(+0) \propto$ $1 / \ln \varepsilon$ for $\varepsilon>0$. It is worth mentioning that in the ferromagnetic case the magnetization is a nonuniversal quantity. In particular, models with different bandwidth cutoffs have values of $\mathcal{M}$ different by $\sim j_{z}^{2}$. To be precise, our mapping is to the bosonized Kondo model (10).

The magnetization jump in the ferromagnetic Kondo model is also related to the problem of a classical Ising chain with inverse-square interaction [18], whose reduced Hamiltonian is $\beta H_{I}=-K \sum_{i>j} s_{i} s_{j} /|i-j|^{2}, s_{i}= \pm 1$. The connection is established by expanding the partition function of the Kondo model in powers of $J_{\perp}$, which is then identified with that of the Ising chain at $K=1 / 4 g$ with additional short-ranged irrelevant interaction that depends on $J_{\perp}$ and bandwidth cutoff. The 1D Ising model with $1 / r^{2}$ interaction has a finite-temperature phase transition [18]. Below the transition temperature, or when $K>K_{c}$, the Ising spins are ordered with a nonvanishing order parameter $\Psi \equiv K\left\langle s_{i}\right\rangle^{2}$. The order parameter vanishes discontinuously as the coupling $K$ is reduced through the $K_{c}$, a 1D analog of the universal jump at the KosterlitzThouless transition. It has been shown rigorously that $\Psi \geq 1 / 2$ in the ordered phase [19]. Although unproved rigorously yet, it is believed that $\Psi=1 / 2$ at $K=K_{c}$, which has been confirmed recently by a large-scale numerical simulation [20]. This means that $\mathcal{M}(-0)-\mathcal{M}(+0)=\left\langle s_{i}\right\rangle=\sqrt{2 g}$, in agreement with our analysis. Note that the external parameter driving the phase transition is $K$ in the Ising model whereas it is $\Gamma / D$ in our model of a level coupled to a $1 \mathrm{D}$ lead.

Thus far, we have ignored Coulomb repulsion between the resonant level and the 1D lead. Assuming that the interaction is short ranged due to screening effects, we may model it by $H_{u}=u S^{z} \frac{d \varphi(0)}{d x}$. Since the unitary transformation gives $U_{2}^{\dagger} H_{u} U_{2}=H_{u}+$ const, the effect of the Coulomb repulsion is to change the Kondo coupling, $J_{z} \rightarrow$ $J_{z}+2 \pi u$. This gives rise to a shift of the phase boundary in Fig. 1 toward smaller $g$. In particular, at $t \rightarrow 0$ the phase boundary is at $g=\frac{1}{2}\left(1+\frac{u}{\hbar v}\right)^{-2}$. Physically, this shift is due to Mahan's excitonic effect [1] that enhances tunneling probability.

It was recently demonstrated [21,22] that the charge of a quantum dot coupled to a Fermi-liquid lead can be measured with the aid of an electrometer based on a single electron transistor. To test our theoretical predictions, one could perform a similar experiment with a lead in the fractional quantum Hall regime. A small dot coupled to the edge state in the lead would then play the role of the resonant level. In this setup, one can vary the parameter $g$ of the chiral Luttinger liquid in the edge state by adjusting the magnetic field. The strength of the coupling of the dot to the edge state in a GaAs heterostructure can be tuned by changing the appropriate gate voltage.
In conclusion, we have shown that, in the region of parameters shown in the phase diagram [Fig. 1], the occupation probability $n(\varepsilon)$ of a resonant level of energy $\varepsilon$ coupled to a Luttinger-liquid lead is a discontinuous function of $\varepsilon$. The height of the discontinuity in $n(\varepsilon)$ at $\varepsilon=0$ reaches $\sqrt{2 g}$ at the phase boundary. This picture is supported by mapping to a Kondo model, and interesting connections can also be made to the problems of the dissipative two-level system and the classical Ising chain with inverse-square interaction.

We are grateful to $\mathrm{S}$. Katsumoto for valuable discussions and to the Aspen Center for Physics where part of the work was performed. K. A. M. acknowledges the support of the Sloan Foundation, the Japan Society for the Promotion of Science, and NSF Grant No. DMR-9974435.

[1] For a review, see, e.g., G. D. Mahan, Many-Particle Physics (Kluwer, New York, 2000), 3rd ed.

[2] A. M. Chang, L. N. Pfeiffer, and K. W. West, Phys. Rev. Lett. 77, 2538 (1996).

[3] M. Bokrath et al., Nature (London) 397, 598 (1999).

[4] Z. Yao et al., Nature (London) 402, 273 (1999).

[5] O. M. Auslaender et al., Phys. Rev. Lett. 84, 1764 (1999).

[6] M. Grayson et al., Phys. Rev. Lett. 86, 2645 (2001).

[7] C. L. Kane and M. P. A. Fisher, Phys. Rev. B 46, 15233 (1992)

[8] C. de C. Chamon and X. G. Wen, Phys. Rev. Lett. 70, 2605 (1993).

[9] A. Furusaki and N. Nagaosa, Phys. Rev. B 47, 3827 (1993); A. Furusaki, Phys. Rev. B 57, 7141 (1998).

[10] X.-G. Wen, Int. J. Mod. Phys. B 6, 1711 (1992).

[11] See, e.g., K. A. Matveev, Phys. Rev. B 51, 1743 (1995).

[12] The Hamiltonian (8) describing nonchiral bosons can be converted by a transformation $\varphi(x)=$ $\sqrt{g} \phi(|x|)+\operatorname{sgn}(x) \theta(|x|) / \sqrt{g}$ to a chiral form similar to Eq. (2).

[13] In the presence of spins, the arguments leading to our main conclusions can still be applied to an Anderson impurity coupled to a semi-infinite Luttinger liquid. The results will be reported elsewhere.

[14] A. J. Leggett et al., Rev. Mod. Phys. 59, 1 (1987).

[15] The second-order result (6) is also found in P. Cedraschi and M. Büttiker, Ann. Phys. (N.Y.) 289, 1 (2001).

[16] S. Chakravarty, Phys. Rev. Lett. 49, 681 (1982).

[17] A. M. Tsvelick and P. B. Wiegmann, Adv. Phys. 32, 453 (1983); N. Andrei, K. Furuya, and J. H. Lowenstein, Rev. Mod. Phys. 55, 331 (1983).

[18] P. W. Anderson and G. Yuval, J. Phys. C 4, 607 (1971).

[19] M. Aizenman, J. T. Chayes, L. Chayes, and C. M. Newman, J. Stat. Phys. 50, 1 (1988); J. Z. Imbrie and C. M. Newman, Commun. Math. Phys. 118, 303 (1988).

[20] E. Luijten and H. Meßingfeld, Phys. Rev. Lett. 86, 5305 (2001).

[21] D. Berman et al., Phys. Rev. Lett. 82, 161 (1999).

[22] D. S. Duncan et al., Appl. Phys. Lett. 74, 1045 (1999). 